\title{
Choix de localisation et mobilisation des ressources dans la création d'entreprises innovantes.
}

\begin{abstract}
Résumé
La création d'entreprises innovantes est censée constituer un des moteurs du développement des systèmes locaux d'innovation, mais elle a rarement été étudiée sous l'angle spatial. Les entreprises innovantes tendent-elles à se regrouper dans l'espace ? Comment les fondateurs choisissent-ils leurs lieux d'implantations? Comment se déploient dans l'espace les ressources mobilisées par les fondateurs ? Cet article fait le point sur ces questions à partir d'un corpus d'histoires de créations d'entreprises constituées sur la base d'entretiens croisés et d'analyse documentaire. L'accent sera en particulier mis sur les choix de localisation et les logiques de mobilisation de ressources dans leur dimension spatiale.
\end{abstract}

Mots-clés : création d'entreprises ; réseaux sociaux ; innovation

\section{Localization choices et resources mobilizing in the creation of innovative new companies}

\begin{abstract}
Creation of innovative companies is supposed to be one of the driving forces of local innovation systems development, but it has not been often studied from a spatial perspective. Do innovative companies tend to geographically concentrate ? How do founders choose the place where they settle their new company? Where are located the resources that founders use ? This article answers these questions on the basis of a corpus of companies creations stories that are based on cross interviews and documentary analysis. We will especially focus on localisation choices and logics of resources mobilizing, from a spatial perspective.
\end{abstract}

Keywords : start-ups ; social networks, innovation 


\section{Introduction}

La création d'entreprises est un moteur majeur de la constitution et du développement des systèmes productifs locaux ${ }^{1}$. Lorsqu'il s'agit de systèmes locaux fondés sur les activités de recherche et développement et sur l'innovation, les nouvelles entreprises se développent sur la base d'innovations techniques ou organisationnelles. Empiriquement, on sait que les entreprises innovantes tendent à s'agglomérer spatialement. Quels sont les mécanismes de cette tendance ? Comment s'effectuent les choix de localisation? Ces entreprises naissent-elle plus facilement dans certains contextes et pourquoi ? Pour répondre à ces questions, nous nous appuierons sur une recherche initiée dans le cadre des programmes « Logiques spatiales de l'innovation $»^{2}$ et «Systèmes locaux de compétences » de la Région Midi-Pyrénées. Cette recherche porte sur les créations d'entreprises innovantes (Grossetti, Beslay, Barthe, 2006). Cette recherche est encore en cours, mais nous présentons ici les résultats acquis dès à présent sur la dimension spatiale du processus de création des entreprises de ce type.

\section{La constitution d'un corpus de cas}

La création d'une entreprise est un processus complexe qui fait intervenir de nombreux acteurs et surtout des niveaux d'action différents. Dans ce processus, le ou les fondateurs, considérés comme des acteurs individuels, donnent naissance à un acteur collectif, une organisation, en mobilisant des ressources et en s'appuyant aussi bien sur des individus que sur des ressources publiques ou des organisations existantes, parfois sur un milieu plus global ou un marché constitué. Chacune de ces entités a une histoire, et ces histoires s'entremêlent dans le processus d'où émerge l'histoire spécifique de la nouvelle entreprise. Dans ces histoires, on peut distinguer des séquences, plus ou moins brèves ou longues selon le découpage que l'on opère. Autrement dit, nous ne faisons pas une étude des entrepreneurs, mais bien une étude des processus de création des entreprises.

Ce processus, nous l'étudions en cherchant à obtenir une narration «contrôlée », qui ne soit pas limitée par le point de vue d'un interlocuteur unique, mais qui ne soit pas non plus une histoire extensive comme pourraient en produire des historiens ou des anthropologues. Il s'agit en effet de constituer un corpus d'histoires suffisamment important pour repérer des régularités et donc gérer en conséquence le temps de travail consacré à chaque histoire. Nous avons donc choisi de réaliser des entretiens multiples (de 2 à 5 selon les cas), complétés par des documents (sites web, documents comptables lorsqu'ils sont accessibles). Les entreprises ont été sélectionnées pour leur caractère innovant selon plusieurs critères: présence ou passage en pépinière, bénéficiaire d'aides à l'innovation, essaimage à partir d'un laboratoire, etc. Sur la base des histoires, nous pouvons étudier plusieurs niveaux d'action : fondateurs, entreprises, relations, mais aussi ce que nous avons appelé des situations de mobilisation de ressources.

Nous avons sélectionné les entreprises que nous avons étudiées à partir des annuaires des pépinières ou incubateurs, et des bénéficiaires d'aides à l'innovation. Nous avons reconstitué

\footnotetext{
${ }^{1}$ Nous utilisons ici ce terme comme expression générique recouvrant l'ensemble des concentrations d'activités marquées par des relations locales de concurrence / coopération et désignées par les termes de «clusters », « technopoles », «districts », «système productifs locaux», etc. Voir (Grossetti, 2004) pour une analyse d'ensemble de ces situations.

${ }^{2}$ Il s'agit d'un programme de la Maison des Sciences de l'Homme d'Aquitaine financé par le Ministère de l’Enseignement Supérieur et la Région Aquitaine.
} 
54 histoires d'entreprises situées à Toulouse (34), Castres (7), Tarbes (5), Decazeville (1), Bordeaux (2), Grenoble (1), Martre-Tolosane (2), Saint-Lizier (1), Montauban (1). 22 d'entre elles sont propriétaires d'au moins un brevet. 28 ont reçu des aides de l'ANVAR, 11 ont été lauréates du concours national de l'innovation. 31 sont ou sont passées en pépinière, 16 en incubateur. 29 sont dans le secteur de l'informatique ou de l'électronique, 13 dans les domaines des biotechnologies (prises au sens large), de la chimie et de la pharmacie, 12 étant dans les domaines de la mécanique ou des matériaux.

\section{Collectif de création et proximités dans la période initiale}

La création d'entreprise n'est qu'exceptionnellement un acte solitaire. Elle implique souvent plusieurs fondateurs dont certains n'ont aucun lien formel avec l'entreprise. Par exemple, dans l'une de nos histoires, un professeur dans une école d'ingénieurs a mis au point une technologie avec deux de ses étudiants de thèse. Il a ensuite suscité la création d'une entreprise autour de cette technologie en trouvant (par les médias) un financeur et PDG qui était un ancien cadre d'un grand groupe. L'entreprise existe donc sans son fondateur principal, qui a préféré rester dans son emploi d'enseignant et n'a pas pris part au capital. Dans un autre histoire, l'un des initiateurs de l'entreprise est membre d'une société cliente, ce qui l'a amené, afin d'éviter un conflit d'intérêt, à ne pas entrer au capital dans les premières années. D'autres fondateurs apparaissent au capital mais ne sont pas membres de la nouvelle société : c'est le cas d'un chercheur du CNRS, détenteur d'un brevet, qui a construit la société développant un produit sur la base du brevet, sans quitter l'organisme dans un premier temps, mais en demandant à son épouse de prendre la direction de la nouvelle entreprise. Enfin, évidemment, de nombreux fondateurs sont membres de l'entreprise, où ils occupent des postes de directeur général, gérant, directeur technique, directeur commercial, etc. Nous avons défini comme fondateur toute personne ayant contribué au montage de la société durant au moins la moitié de la période de fondation, sans que cela soit son métier. Sur la base de cette définition, nous en avons trouvé une moyenne de 2 par entreprise, le maximum rencontré s'établissant à 5.15 entreprises seulement $(37 \%)^{3}$ ont un seul fondateur, ce qui signifie que $63 \%$ en ont au moins 2. Cette dimension collective du processus de création d'entreprise entre en contradiction avec le modèle de l'entrepreneur solitaire, sur lequel les institutions tendent à ajuster leurs procédures, en demandant systématiquement que l'un des fondateurs se mette en avant et endosse le rôle de l'entrepreneur.

Dans la plupart des cas, on observe donc l'existence d'une sorte de "collectif de création" qui se met en place avant même le dépôt des statuts (ce collectif connaîtra ultérieurement des destinées variées). Ainsi les créateurs cherchent-ils à valider une idée initiale auprès de proches, qui seront le plus souvent d'anciens collègues, des amis ou des membres de l'entourage familial. Le cas de VIOLON ${ }^{4}$ est particulièrement exemplaire de cette situation. Le fondateur initial, en situation de recherche d'emploi, reprend contact avec un ancien employeur connu dans une autre structure. Celui-ci le met en relation avec un ancien collègue de cette même structure. C'est ensemble que les trois hommes vont discuter, faire évoluer et surtout valider une idée initiale. Cette "réunion" permettra également de dégager les premières ressources (local, matériel, compétences techniques, prémisses de partenariats), qui permettront demain au projet de voir le jour. Dans cet exemple l'ensemble des ressources mobilisées répond à une triple exigence de proximité. Proximité géographique tout d'abord.

\footnotetext{
${ }^{3}$ Comptages effectués sur 39 cas ayant pu être codés, les 15 autres ne l'étant pas encore au moment de la rédaction de ce texte. Ces 15 cas sont utilisés pour l'analyse qualitative.

${ }^{4}$ Pour des raisons de confidentialité, les noms des entreprises sont fictifs.
} 
Toutes les ressources initiales sont concentrées dans l'agglomération de résidence des fondateurs. Proximité professionnelle ensuite: ce sont d'anciens collègues se situant à différents niveaux hiérarchiques d'une entreprise qui se sont tous croisé à un moment de leurs histoires personnelles. Ils partagent donc un passé commun et savent situer leurs différents apports personnels, notamment sur le plan technique. Proximité relationnelle ensuite, dans la mesure où les trois hommes se connaissent depuis longtemps et où une certaine estime (ou confiance) semble exister entre eux.

Cette triple proximité est renforcée dans les cas de créations d'entreprises fortement adossées à un laboratoire de recherche ou dans les cas d'entreprises créées dans le cadre de la loi sur l'innovation. Là, l'interconnaissance des protagonistes est encore plus forte (fréquentes relations entre thésards et directeurs de recherche par exemple). Les membres du collectif se connaissaient déjà et les compétences techniques des uns et des autres étaient déjà identifiées et reconnues. Comme dans le cas de VIOLON, la concentration géographiquement très localisée des ressources et l'existence d'un passé commun permet de dégager rapidement un petit volume de ressources initiales (le plus souvent locaux et compétences techniques) qui permettront au moins de préparer et de peaufiner le projet.

De la même manière, dans les cas d'essaimage ou de reconversion, les fondateurs connaissent déjà de par leur expérience professionnelle ceux qui seront demain leurs partenaires ou leurs associés. Et même si, dans un petit nombre de cas, l'un des fondateurs voyait dans le projet de création une possibilité de départ, de nombreux facteurs contribuent à générer une inertie qui fait que l'on va créer l'entreprise là où les premières ressources sont immédiatement disponibles. Ainsi dans le cas de l'entreprise CLARINETTE, Laurent aurait aimé changer de région, voire créer l'entreprise à l'étranger, "moi je ne pensais pas forcément rester ici, je pensais aller en Espagne ou en Suisse... Les petits étaient petits, et ma famille, l'expérience de la Suisse ou l'Espagne, ça les intéressait... J'aurais aimé une expérience autre, mais bon, ça c'est passé comme ça». En effet, Gilbert, associé de Laurent était peu mobile, avec trois enfants scolarisés. Et comme Laurent, de par son activité professionnelle, «connaissait les gens qui s'occupaient de l'économie au Conseil général, il est maire, il m'a proposé des facilités d'installation et je me suis laisser séduire [...].». Pour d'autres, comme Vangelis, fondateur de FLUTE, il est tout simplement impensable de quitter la région où l'on s'est installé et où l'on est si bien. Mais pas question pour autant de créer sans la garantie de l'existence de ressources qui rendront le projet viable. Vangelis va créer sa société dans le cadre d'une situation d'essaimage, son employeur précédent venant d'abandonner l'activité dans laquelle notre fondateur s'était spécialisé. La première ressource recherchée est celle de l'existence de débouchés. Dans son cas : "On a été appuyés par des clients. [...] Du point de vue famille aussi, j'ai été appuyé. [...] Du temps qu'il était là, je sentais que [mon père] voulait que je monte quelque chose. De tout temps il a insisté. [...] après j'avais les collègues qui étaient intéressés [ils deviendront employés et entreront au capital de l'entreprise]. Des fournisseurs semblent l'avoir également soutenu dans son projet. Vangelis se tourne tout naturellement vers le député au Conseil Général à xxxxx qu'il connaissait déjà par implication personnelle dans la vie politique, d'autant que son père a été maire de $\mathrm{xxxxx}$ : "Après on est allés voir le sous-préfet aussi, je pense. Parce qu'on cherchait où c'est qu'on pouvait $s$ 'implanter ». Ce sont ces contacts qui l'orienteront vers la pépinière locale qui lui proposera ses premiers locaux, condition sine qua non à la création de l'entreprise.

Le cas de Vangelis est un peu caricatural, mais il a le mérite de rappeler que la création d'entreprise peut être assimilée à une tentative de résolution d'un problème comprenant de multiples inconnues. L'existence de ressources initiales constitue l'une de ces inconnues. Par 
conséquent la tentation est peut-être de créer une entreprise là où il y a le moins d'inconnues, c'est-à-dire là où il existe un ensemble de ressources préexistantes et déjà identifiées par les porteurs du projet. Ce n'est pas tant le projet d'entreprise qui dicte le choix du l'endroit où l'on va créer, que l'identification et la localisation des ressources initiales qui permet de penser que l'acte de création est envisageable comme une hypothèse réaliste. Ceci se traduit le plus souvent une forte concentration géographique (on se situe le plus souvent au niveau de l'agglomération) de ces mêmes ressources.

Sur le plan des ressources humaines (compétences techniques initiales, partenaires associés, premiers conseillers) tout semble se passer comme si les fondateurs essayaient de se doter de la corde de rappel la plus solide et la plus courte qui soit. On cherche près de soi les meilleures assurances auprès du "collectif de création" avec qui on partage une certaine proximité géographique, professionnelles et personnelle. Ce collectif semble agit comme une sorte de garde fou dans l'acte de création. Le cas de SAXOPHONE en est particulièrement exemplaire. Le fondateur avait été déçu de voir un brevet sur des agents anticancéreux potentiels insuffisamment exploité. L'un de ses amis chercheur dans la même spécialité (géographiquement distant) l'encouragera à créer son entreprise. Le "collectif de création" sera constitué de la femme du fondateur, qui assurera la direction de l'entreprise, son fils, qui amènera des compétences organisationnelles, son ami qui lui prodiguera conseils et assistance, les 3 co-auteurs du brevet, un professeur, membre de l'équipe du fondateur, un polytechnicien à la fois ami du fondateur et de son ami conseiller. Le "collectif de création" constitue un petit capital et le fondateur se lance avec l'aide de son ami conseiller à la recherche d'un premier tour de "levée de fonds ». Ils montent un business plan avec l'aide d'un cabinet d'experts-comptables («un cousin de [l'une des co-auteur du brevet] », puis, sur les conseils du fils, font appel à l'antenne locale d'un cabinet.

Cette notion d'assurance par la proximité est d'ailleurs confirmée de manière périphérique dans un petit nombre de cas d'entreprises créées alors que leur clientèle existait déjà (ce qui s'observe dans certains cas d'essaimage). Il arrive que, dans de tels cas, les clients expriment le besoin de disposer de sous-traitants géographiquement proches, la raison invoquée étant une espérance de réactivité et de contrôle de qualité dans les livraisons. Pour [le client], [le projet d'entreprise] représentait l'opportunité d'avoir « un sous-traitant devant sa porte ». Cet argument de la proximité pour un équipementier qui travaille en flux tendu était un gage de souplesse et de réactivité. De fait, "ils nous ont testé, le $1^{e r}$ marché qu'ils nous ont donné, ce sont des rondelles isolantes pour les bougies de préchauffage. La difficulté était d'en fabriquer 1.500.000 par mois. On a fait tout ce qu'il fallait au niveau des contrôles ».

Toutefois le "collectif de création" ne maîtrise le plus souvent pas la totalité des ressources nécessaires à l'émergence d'une entreprise. C'est notamment le cas pour les conseils ou l'assistance à la création d'entreprise. La plupart du temps ces ressources sont accessibles par le biais des incubateurs ou des pépinières d'entreprise, ou encore sur les conseils d'amis appartenant au noyau des fondateurs. Dans notre étude, l'échantillon est pour l'essentiel composé de structures qui ont émergé en milieu urbain ou à la proximité de petites villes (certains cas d'essaimage). Les ressources permettant d'accéder aux conseils ou à l'assistance recherchée seront par conséquent la plupart du temps elles-mêmes situées dans l'espace de l'agglomération. Dans notre population d'étude, les contre-exemples sont extrêmement rares.

Il faut cependant noter le cas particulier d'une ressource spécifique : les financements. En fait tout dépend, de la nature du projet d'entreprise d'une part, et de la stratégie développée par les fondateurs d'autre part. Deux cas peuvent se présenter. Soit les créateurs n'ont besoin que d'un 
fonds de démarrage limité (il s'agira de ne disposer que de quelques fonds permettant de tester la faisabilité du projet, par exemple de développer un peu de R\&D en attendant d'atteindre l'autofinancement). Dans ce cas, les ressources financières initiales seront recherchées dans les mêmes conditions de proximité que les autres ressources (autofinancement, allocation chômage pour les anciens étudiants thésards, prêts ou dons familiaux, investissements sur le projet d'entreprise par des collègues, des amis, d'anciens employeurs), ou au travers de diverses aides de l'ANVAR, par exemple. Soit le projet d'entreprise suppose de lever immédiatement des fonds. Il faut alors organiser très tôt un tour de table. C'est souvent le cas des entreprises de biotechnologie ou d'électronique, par opposition aux entreprises de type informatique bancaire ou scientifique. On observera dans de tels cas les mêmes conditions de création, d'accès et de localisation de ces ressources (proximité professionnelle et relationnelle) mais la notion de proximité géographique peut être moins importante. Le cas de [BASSE] en est un bon exemple. Comme Les fondateurs souhaitent valoriser une découverte faite en 1998. Pour cela ils souhaitent créer une entreprise dans le cadre de la loi sur l'innovation de 1999. Ils cherchent un investisseur externe, également Parisien. Un collègue distant (il est à Paris) leur permet d'accéder à un investisseur potentiel. Pour diverses raisons, l'opération n'aboutira pas immédiatement. Il faudra attendre quelques années (2001) pour que l'investisseur pressenti se constitue en fonds d'amorçage privé dédié au lancement d'entreprises de biotechnologies et... investisse. Ce dernier deviendra au passage le PDG de la nouvelle entreprise, même s'il vit et agit essentiellement aux Etats-Unis. Dans ces cas un peu particuliers, la plupart des ressources initiales vont êtres situées au niveau de l'agglomération (compétences techniques, humaines, locaux, etc.). L'argent peut avoir une autre provenance, mais la ressource sera malgré tout accessible par le biais de d'un collectif de création professionnellement et relationnellement proche, et géographiquement très localisé à l'endroit où les gens se sont rencontrés notamment sur le plan professionnel.

Il existe toutefois une série de cas plus rares : les créations d'entreprises qui sont partiellement conditionnées par la réalisation d'un tour de table préalable. Il s'agira le plus souvent d'entreprises de biotechnologie ou d'entreprises High Tech qui veulent à la fois financer leurs R\&D et/ou la mise en production des résultats de leur R\&D [situation initiale de BASSE]. De telles situations peuvent se trouver quand le fondateur estime être, par son passé professionnel, proche du milieu des financiers, ou quand les fondateurs ne sont pas en mesure d'assumer les coûts et les risques d'un tel engagement. Quand de tels cas se présentent la maturation du projet peut être un peu plus longue que dans les autres cas (jusqu'à ce qu'un investisseur se présente, ce qui peut prendre quelques années). L'accès à la ressource peut également être moins géographiquement localisé que dans les autres cas, puisque l'argent de la création n'est qu'en partie l'argent propre du fondateur ou du "collectif de création".

\section{Les « choix » de localisation}

De nombreux travaux sont consacrés à la question des choix de localisation des entreprises et en particulier des start-ups (Zucker, Darby et Armstrong, 1994), le problème majeur étant souvent d'évaluer leur tendance à rester sur les lieux de leur création ou au contraire à rechercher les localisations les meilleures (Stam, 2006). Nous ne nous intéresserons ici qu'à la question de leur localisation initiale.

Les «choix de localisation » peuvent être considérés à plusieurs niveaux. En premier lieu, il s'agit de l'implantation de l'entreprise, identifiée à son siège social et à son établissement principal, sur un territoire à l'échelle d'une agglomération ou d'un bassin d'emploi : pourquoi 
s'installer dans l'agglomération toulousaine plutôt qu'à Grenoble, Bordeaux, Paris ou même à l'étranger, à Figeac plutôt qu'à Rodez, Albi ou Gourdon ? Se pose ensuite la question de l'installation précise dans telle ou telle commune ou partie d'une agglomération ou d'un bassin d'emploi, sur telle zone industrielle, voire tel ou tel quartier. A ces deux niveaux, plusieurs questions se posent. La localisation des entreprises est-elle le produit d'une stratégie raisonnée, au regard des marchés ou des partenariats engagés? Quel est le poids des politiques industrielles locales et des dispositifs mis en œuvre pour accueillir ou aider la création d'entreprises? Quelle est l'importance des relations sociales et notamment des contraintes familiales dans ces choix de localisation?

En fait, les «choix » de localisation, aux deux niveaux envisagés de l'agglomération ou du bassin d'emploi, ne renvoient pas tellement à des stratégies économiques ou même industrielles, qu'il s'agisse de capter des aides financières ou de se rapprocher des marchés ou des partenaires. L'implantation est plutôt adossée aux stratégies familiales et aux choix de vie. Ensuite, l'accès à des locaux mobilise très fortement les relations sociales et les dispositifs d'accompagnement et d'aide à la création et à l'installation d'entreprises.

\subsection{La localisation des entreprises : le poids des logiques familiales et des projets de vie}

Pour le premier niveau, on peut dire que la question des choix de localisation ne se pose que très rarement. Dans la plupart des cas, la nouvelle entreprise est installée à proximité des lieux de vie et d'activité du ou des créateurs. Dans plus des deux tiers des entreprises étudiées, au moins l'un des créateurs était originaire de la région. Leur trajectoire professionnelle a pu les amener à quitter la région, plus ou moins longtemps, mais le plus souvent la création d'entreprise n'est ni le mobile, ni l'alibi du projet de «retour au pays ». Ainsi, jean-Louis a fait une grande partie de sa carrière dans une multinationale américaine dans des postes commerciaux à responsabilité aux Etats Unis, à Singapour, en Libye... La profession de son épouse (hôtesse de l'air) était compatible avec ces mobilités géographiques. Mais, à 36 ans, à un tournant de sa carrière dans l'entreprise, les enfants grandissant, il ressent l'envie de rentrer au pays, «c'est le moment de rentrer ». Ayant par ailleurs l'envie de se mettre à son compte, sans idée préconçue pour telle ou telle activité, il rencontre, par l'intermédiaire de la CCI, un porteur de projet, qui a des idées mais pas de capitaux, et, avec lui, il crée une petite entreprise de vente d'espace vidéo texte.

A quelques exceptions près, les créateurs étaient installés, travaillaient et/ou habitaient dans l'agglomération ou le bassin d'emploi dans lequel ils ont créé leur entreprise. Rares sont les cas où la création s'est accompagnée (a nécessité) un changement de lieu de vie des créateurs (en tous cas de tous les créateurs).

En fait, la question de la localisation de l'entreprise se pose différemment selon le contexte de la création :

- Lorsqu'il s'agit d'essaimage de laboratoires, il est fréquent que les chercheurs conservent leurs fonctions dans leur laboratoire (ou cherchent à le faire) et tentent d'éviter une trop grande dispersion de leurs activités. Une localisation dans la même agglomération suffit en général à satisfaire cette contrainte. Les partenariats engagés au sein du laboratoire justifient une proximité spatiale, d'autant que les différents acteurs sont déjà installés localement et que rien ne milite vraiment pour une autre localisation. Bertrand, professeur dans une école d'ingénieurs, dirige une équipe spécialisée sur le calcul à haute performance pour des besoins de simulation. Après l'échec d'une entreprise qui voulait commercialiser les résultats de recherche de l'équipe de Bertrand et avait recruté ses deux doctorants, Bertrand décide de se lancer lui-même dans l'aventure de la création 
d'entreprise, profitant de l'opportunité de nouveaux marchés et soucieux du devenir professionnel de ses anciens doctorants. Gérant de l'entreprise, il désire néanmoins conserver son poste d'enseignant. Mais professeur contractuel d'un établissement sous tutelle du Ministère de la Défense, il ne bénéficie pas des dispositions de la Loi sur l'Innovation de 1999. Il demande un congé sans solde, mais ne souhaite pas rompre les liens avec son établissement d'origine.

- Dans les cas d'essaimage ou de reprise d'entreprise existante (en totalité ou en partie), la question de la localisation peut se poser. Mais le plus souvent, les liens avec l'entreprise d'origine, la localisation de la main d'œuvre et l'implantation locale des créateurs font obstacle à d'éventuels projets d'installation dans d'autres sites. C'est le cas de Laurent qui a créé avec Gilbert une entreprise de conception et fabrication de pièces techniques en céramiques pour répondre aux besoins d'un marché que son entreprise d'origine ne voulait pas investir. Dans ce tournant de carrière, Laurent aurait aimé changer de région, voire créer l'entreprise à l'étranger, " moi je ne pensais pas forcément rester ici à Tarbes, je pensais aller en Espagne ou en Suisse... Les petits étaient petits, et ma famille, l'expérience de la Suisse ou l'Espagne, ça les intéressait... J'aurais aimé une expérience autre, mais bon, ça c'est passé comme ça ». Ce n'était pas l'avis de Gilbert, peu mobile, avec trois enfants scolarisés. C'est le cas également d'une entreprise créer par un consortium de groupes coopératifs agricoles pour prendre en charge des fonctions de R\&D. L'entreprise fonctionnant avec des personnels mis à disposition par les coopératives, elle est naturellement localisée à proximité du groupe dont sont issus ses principaux dirigeants.

- Dans le cadre de reconversions industrielles, le maintien de l'activité dans ces territoires en difficulté économique est un des objectifs explicites des dispositifs d'aide à la création d'entreprise. Certaines aides sont conditionnées par la localisation de l'activité. Pour les anciens salariés créateurs d'entreprise, la création est d'ailleurs une solution pour rester vivre là où ils habitaient, c'est aussi participer au re-développement local. Jacques, René et Paul ont créé leur entreprise d'étude et fabrication de pièces techniques en plastique, dans le cadre du dispositif de reconversion mis en place lors de la fermeture de leur entreprise. Dans ce territoire rural, l'annonce de la fermeture de l'entreprise «c'est un peu le ciel qui nous est tombé sur la tête ». Les trois associés, originaires du département, ont peu de perspectives d'emploi local, le tissu industriel étant peu dense et en difficulté. L'idée d'une mobilité géographique «ne nous intéressait pas», notamment du fait de l'activité professionnelle de leurs épouses, dont certaines avaient «une situation intéressante et elle n'était pas prête à le lâcher ». La création d'une entreprise est ainsi la seule solution pour rester vivre sur place.

- Dans les autres cas, que la création soit une solution d'emploi, une opportunité ou un projet, il est rare que la localisation fasse réellement l'objet d'un choix raisonné. Tout se passe comme si la question de la localisation était perçue comme extrêmement secondaire par les créateurs, voire comme allant de soi et ne posant pas question. En fait, l'entreprise est créée là où vivent les créateurs. Si les acteurs n'ont que très rarement des stratégies de localisation pour leur entreprises, il arrive bien plus fréquemment qu'ils en aient pour leur vie familiale. Ce sont d'ailleurs souvent des projets de vie et des stratégies familiales qui expliquent in fine la création ou l'installation d'une nouvelle entreprise dans une agglomération, voire le passage à la création lui-même. Vangélis, électronicien de formation, responsable du bureau d'études d'une entreprise en milieu rural périurbain, perd son emploi suite aux difficultés économiques de son entreprise. Il n'a guère le choix. Il n'y a pas d'entreprise sur le secteur. Il lui faut donc s'expatrier à l'étranger, aller à Toulouse, ou créer son entreprise, ce dont il n'avait jusqu'alors jamais eu l'idée. Désireux 
de rester vivre sur place, il est d'ailleurs conseiller municipal, incité par d'anciens clients, d'anciens collègues et par sa famille, il décide de tenter l'aventure de la création d'entreprise. Mickaël, ingénieur anglais, après une carrière dans des grandes entreprises internationales, souhaitait, à 55 ans, s'installer avec sa famille, pour sa retraite, dans la petite ville de l'Aveyron où il a passé plusieurs séjours de vacances et acheté une résidence secondaire. Désireux de ne pas rester totalement inactif et de «faire quelque chose pour le développement local », il a créé une petite entreprise commercialisant des systèmes d'acquisition de données.

Les créations où la localisation fasse réellement l'objet d'un choix raisonné sur des considérations économiques sont rares (pas plus de trois ou quatre cas dans notre corpus d'une cinquantaine d'entreprises). Nous avons identifié deux cas de figure non exclusifs :

- Des montages impliquant des acteurs dispersés dans l'espace. La localisation peut alors être un enjeu. Ces situations impliquent bien souvent des stratégies de la part des responsables des organismes d'accompagnement ou de la part d'autres personnes impliquées dans les activités de développement local, pour «attirer» les nouvelles entreprises. Pierre, chercheur CNRS dans un laboratoire parisien d'hématologie est originaire de la région Midi-Pyrénées et a fait ses études à Toulouse. Avec deux collègues, il a mis au point une nouvelle méthode de prélèvement de moelle osseuse dont il souhaite exploiter le brevet. Par l'intermédiaire de la belle-mère de son fils, qui travaille à la mairie de Toulouse, il est mis en contact avec Richard, du service de développement industriel de l'agglomération qui apportera un soutien financier et lui présente Michel, ancien commercial d'un groupe pharmaceutique recherchant de projets à développer, qui deviendra associé dans l'entreprise dont il assurera la direction générale. Richard, aidé par Michel, convainc Pierre et ses deux collègues associés d'installer l'entreprise à Toulouse, dans l'incubateur de Midi-Pyrénées. Pierre et ses deux collègues travaillent toujours à Paris et seul Michel est à Toulouse. Pierre fait de fréquents séjours à Toulouse où réside une grande partie de sa famille, dont son fils. L'implantation de l'entreprise renvoie en fait à plusieurs éléments : la rencontre, par relations sociales, d'un associé, l'aide d'une collectivité locale, l'origine géographique du créateur et sa situation familiale.

- L'opportunité de bénéficier d'aides à l'installation. Charles, originaire de la région Auvergne, ancien militaire, technicien de l'armement, est arrivé à Toulouse, après de nombreuses mobilités géographiques, pour créer une agence infographie d'une grande entreprise nationale. Licencié de son entreprise, il décide, avec ses indemnités de licenciement, de créer une société d'étude et d'ingénierie géomatique pour l'aéronautique. Parce qu'il veut «vivre dans le sud», il décide de créer son entreprise à Toulouse. Lors d'un dîner d'affaire organisé par le Conseil régional, il rencontre un élu municipal d'une petite ville du Tarn qui le convainc d'y implanter son entreprise : "je ne savais même pas où c'était. Il m'a dit que j'aurais beaucoup d'intérêt à y venir. Donc, j'ai dit : pourquoi pas, qu'est-ce que vous me proposez? Il m'a proposé un loyer pas cher. Et puis habiter à la campagne, ça me tentait». Charles, qui se définit volontiers comme un «chasseur de primes », s'est laissé convaincre par les aides à l'installation, mais aussi parce que l'implantation correspondait également à son projet de vie.

\subsection{Le choix des locaux : le poids des politiques locales}

$\mathrm{Au}$ second niveau en revanche, les choix sont plus fréquents : l'entreprise va être créée dans l'agglomération de Toulouse (de Bordeaux, Grenoble, Castres, etc.), mais elle peut l'être dans telle ou telle pépinière ou telle ou telle zone d'activité. La question est ici de trouver des locaux. Bien sûr, les situations sont différentes selon la nature de l'activité, l'effectif de 
l'entreprise et de la surface dont elle a besoin. Certains créateurs ont pu démarrer leur activité à leur domicile personnel, avec juste un téléphone et un ordinateur. Mais lorsqu'il y a des salariés et des équipements scientifiques ou industriels, il faut des bureaux et des bâtiments. A ce niveau, les politiques locales et les dispositifs d'aide à la création d'entreprises jouent un rôle important. Nous avons en fait rencontré plusieurs cas de figure :

- La plupart de nos créateurs ont trouvé un premier hébergement en pépinière $(57 \%)$ ou en incubateur $(30 \%)$. Les raisons en sont d'abord financières (pour une jeune entreprise, le coût d'un tel hébergement est modeste), mais relèvent aussi d'une logique d'affichage (de fait, la pépinière confère une sorte de label «Sart-Up High Tech »), et renvoient à la possibilité de bénéficier d'un appui / conseil au développement, de services de secrétariat et, dans le cas des biotechnologies, à l'accès à certains équipements spécifiques. Dans certains cas, l'hébergement en pépinière n'est qu'une boîte au lettre, comme dans le cas de l'une de nos entreprise, créée par des chercheurs qui continuent à travailler dans leur labo mais ont un bureau dans un pépinière pour bénéficier d'une adresse et d'un accueil téléphonique

- Il arrive que l'entreprise, essaimée d'un laboratoire ou d'une entreprise, soit hébergée, au moins dans un premier temps, par la structure d'origine. Six entreprises ont, dans un premier temps, trouvé un tel hébergement. C'est le cas des entreprises filiales R\&D de coopératives agricoles, hébergées dans les locaux du groupe ou d'un des groupes fondateurs. Hubert, chercheur en télécommunication dans un laboratoire CNRS, a créé une entreprise pour valoriser un nouveau modèle de conception et de planification des réseaux de télécommunication, entreprise qui est hébergée par le laboratoire d'origine, dans le cadre d'une convention de deux ans renouvelable. Cet hébergement, outre un coût réduit, permet de bénéficier des équipements informatiques, de la bibliothèque et d'une salle de conférence du laboratoire. Cela permet également de pouvoir accueillir les clients dans un cadre valorisant. Charles, le «chasseur de prime » déjà évoqué, décide, après l'échec et le rachat de sa première entreprise, de créer une nouvelle société pour commercialiser un système d'information géographique avec un nouvel associé. Ils ont investi à titre quasi gratuit les vastes locaux sous-utilisés d'une petite entreprise partenaire dont l'associé était le directeur, et dont Charles est l'un des actionnaires.

- Dans quelques cas encore (5 cas), les entreprises utilisent les structures d'accueil mis en place par les collectivités locales : ateliers relais, zones industrielles ou artisanales, parcs scientifiques. Il s'agit surtout d'entreprise des secteurs mécanique ou matériaux, qui ont besoin de vastes locaux pour héberger un important parc de machines et des salariés. Les locaux sont loués ou construits. Jacques, René et Paul, lorsqu'ils ont créé leur entreprise dans le cadre de la reconversion de leur entreprise d'origine, ont d'abord été accueillis dans un bâtiment / atelier relais mis à leur disposition par la municipalité. Laurent et Gilbert ont construit les bâtiments de leur entreprise de pièces techniques céramiques sur une petite zone industrielle parce que Laurent «connaissait un maire qui m'a proposé des facilités d'installation et je me suis laissé séduire ».

- Enfin, plus rares encore (trois) sont les cas d'installation dans des locaux trouvés sur le marché immobilier privé. Il s'agit d'entreprises de service avec peu de personnel et d'équipements qui louent des bureaux en centre ville.

Il existe donc une sorte de «marché » du logement des entreprises en création qui met en concurrence les structures d'accueil (pépinières, zones industrielles...) d'un côté et les entreprises de l'autre. Mais ce marché est fortement structuré par les dispositifs publics qui décident de l'implantation et donc de la localisation de ces structures. Ce «marché » est également fortement structuré par les relations sociales. Nous avons relevé l'importance des 
réseaux sociaux dans l'accès à une pépinière, un atelier relais, une zone industrielle... En général, le créateur ou l'un des créateur entre en contact, par connaissance, avec un élu, le directeur d'une pépinière, un agent de développement local... qui propose l'accueil et en présente les avantages. Il n'y a que rarement un «effet catalogue » dans lequel les créateurs feraient en quelque sorte leur marché parmi un éventail de propositions ouvertes ; il y a plutôt des opportunités d'hébergement qui se construisent au travers des rencontres entre les acteurs. Dans la plupart des cas également, l'entrée dans les locaux ne résulte pas d'une relation purement marchande, mais résulte de choix réciproques qui s'expriment notamment par un acte formel de candidature et des critères d'éligibilité.

Enfin, mais à un degré moindre que pour le choix de la localisation de l'entreprise, le choix des locaux est contraint par les lieux de vie des salariés et du ou des créateur(s) (et de leur famille). Ainsi, lorsque Pierre a repris le service R\&D de son entreprise d'origine pour développer de nouveaux produits, il a construit de nouveaux locaux en face de l'ancienne entreprise, pour tenir compte du fait que les salariés habitaient dans un rayon choisi en fonction de la localisation de l'entreprise, anticipant les difficultés que certains pourraient rencontrer si l'entreprise se re-localisait ailleurs.

\section{Premières heures de vol}

Une fois que les entreprises ont été formellement créées et avant qu'elles n'aient atteint leur vitesse de croisière, nous constatons trois situations.

La première d'entre elle est d'abord de la "pérennisation" des ressources locales qui ont été initialement convoquées. Grossièrement ceux qui ont participé à l'élaboration de l'idée et qui, dans un certain nombre de cas, forment le cortège des premiers associés continuent de jouer un rôle de conseiller. La plupart du temps ces personnes qui avaient déjà amené des compétences techniques ou des expertises... continuent de les amener et participent par exemple activement à des projets de R\&D. Suivant les cas il pourra s'agir de compétences de gestion, mais, pour des sociétés adossées à des laboratoires de recherche et issues de la loi sur l'innovation de 1999, il pourra également s'agir de compétences scientifiques (ce qui est flagrant dans le cas des entreprises de biotechnologies, où les fondateurs jouent fréquemment les rôles de conseillers scientifiques). Qu'il s'agisse de compétences techniques, scientifiques ou organisationnelles, ces ressources qui avaient été identifiées et mobilisées localement à de rares exceptions près... demeurent naturellement locales.

La seconde situation renvoie plus directement aux ressources logistiques nécessaires aux entreprises en cours de développement. Le "collectif de création" est moins autodidacte et va avoir tendance à rechercher des compétences externes dans divers métiers. Les comptables, avocats et banquiers vont ainsi être recherchés au-delà des premières indications qui souvent fournies par les incubateurs ou les pépinières. Le cas de XYLOPHONE en est exemplaire : la société est issue d'un essaimage et, dans la phase de maturation de son projet, a été entièrement assistée par l'entreprise mère. Le moment de son démarrage effectif est marqué par une rupture avec les "prestataires de services" antérieurs. Pourtant ces compétences ne sont pas recherchées très loin, au contraire. Là encore on tend à rechercher une double proximité relationnelle et géographique. Dans le cas de VIOLON l'avocat est celui qui avait défendu le fondateur aux prud'hommes à l'occasion d'un contentieux antérieur à la création de l'entreprise. Pour XYLOPHONE le fondateur va prendre pour comptable un professionnel connu de l'un de ses associé et pour avocat un cabinet choisi un peu au hasard et situé à 4 kilomètres de l'entreprise. Dans le cas de CYMBALE, l'expert comptable est identifié par la 
femme du fondateur, elle-même comptable. Ce fondateur, au demeurant très pragmatique, déclarait d'ailleurs : ça me permettait d'avoir du conseil à la maison. Il n'y a pas de petit profit. Dans le cas de VIOLONCELLE c'est une ancienne petite amie qui indiquera un comptable également installé à proximité de l'entreprise. Notons au passage que ce cas de figure est loin d'être rare et que, bien souvent, l'avocat est identifié grâce à l'expert comptable qui a lui-même été identifié par l'intermédiaire d'un proche du fondateur ou du collectif de création. Dans la majorité des cas, l'échelle géographique est celle de l'agglomération. On trouve toutefois quelques rares contre exemples : il s'agit le plus souvent de sociétés, par exemple de biotechnologie, dont l'un des associés ayant une envergure nationale ou internationale va "amener" les prestataires de services avec lesquels il avait auparavant l'habitude de travailler.

D'autres compétences sont également rapidement recherchées : ce sont les personnels qui seront les premiers employés ou éventuellement les premiers stagiaires de l'entreprise nouvellement créée. Là encore les mêmes tendances doivent être constatées : les premiers recrutements (le plus souvent des profils techniques) se font par relation de travail pour ceux qui sont en contact étroit avec des laboratoires de recherches ou des projets européens, soit au travers des formations avec lesquelles les membres du collectif de création ont très souvent gardé le contact. Le fait par exemple de participer en tant que professionnel à un Jury de thèse peut ainsi contribuer à l'identification d'un profil recherché. Le cas de HARPE est particulièrement révélateur de cela. Le gérant de l'entreprise avait été recruté car c'était un ami de la famille de l'un des principaux acteurs du collectif de création. Le directeur de la recherche travaillait en tant que chef de projet dans l'ancienne société du principal associé (et financeur). Le principal chercheur avait effectué sa thèse sous la direction du cofondateur dans le laboratoire du principal fondateur. L'ingénieur travaillait dans l'unité initiale du second associé. Dans ce cas particulier, deux compétences ont été ramenées sur Toulouse, mais ce cas est assez exceptionnel. La plupart du temps les ressources en personnel sont identifiées sur place. Dans les cas d'essaimage on retrouve naturellement cette tendance à travailler ou à retravailler avec des personnes que l'on connaissait déjà. Notons que les aides institutionnelles au démarrage tendent à s'amoindrir à ce niveau, alors que les aides institutionnelles à l'embauche (prise en charge d'une partie des salaires) ou à l'accès à différentes prestations de services ont tendance à augmenter. Les ressources recherchées sont toujours très locales, mais leurs formes se modifient. De la même manière, dans les cas de reconversion, les ressources en personnels sont identifiées au travers des multiples dispositifs mis en œuvre, comme dans le cas de VIELLE : «je ne les connaissais pas spécialement [les salariés]. VM nous avait donné une liste et on en a coché 3 ou 4. On leur a bien expliqué qu'ils n'étaient plus à VM, mais dans une petite entreprise ». De la même manière, les stagiaires sont identifiés soit au travers des formations avec les quelles les fondateurs sont restés en relation, soit au travers d'organismes locaux de formations (AFPA ou GRETA par exemple), qui prospectent sur leur zone.

Dernier type de ressources associées à cette situation : les ressources matérielles. Il s'agira le plus souvent des locaux simples, ou des locaux impliquant des aménagements particuliers (salles blanches, paillasses, animaleries, etc.). Comme pour les autres ressources, on observe les mêmes tendances. Les locaux restent près des pépinières. Le cas de XYLOPHONE en est un exemple presque caricatural : l'entreprise occupera les mêmes locaux que ceux occupés quelques années auparavant par l'entreprise mère qui l'aura essaimée. Dans certains cas, on observe un léger éloignement, bien que l'on reste au niveau de l'agglomération. Quelques structures profiteront ainsi de certaines aides à la réindustrialisation des sites dévastés par l'explosion d'AZF. Les entreprises de biotechnologies qui auront émergé dans les pépinières 
s'installent dans de nouveaux locaux spécifiques qui leurs sont dédiés, mais toujours à proximité des pépinières. Quand il y a reconversion d'un site industriel, il arrive que des politiques de revalorisation ancrent les nouvelles sociétés sur les zones en réhabilitation. Ce fut le cas pour VIELLE. D'abord logée dans un atelier relais, l'entreprise s'est installée, au bout d'un an, dans des bâtiments laissés vacants par le groupe d'origine et acquis dans des conditions avantageuses, «ils m'ont fait beaucoup de facilités pour que je devienne propriétaire. Ils $m$ 'ont donné un bon coup de main pour démarrer $»$. Le groupe d'origne a également soutenu la création, en prenant à sa charge la fourniture d'électricité et par du prêt de matériel en cas de besoin. En bref, il est extrêmement rare que le démarrage proprement dit des entreprises se traduise par une importante mobilité géographique. Les ressources mobilisées restent toujours aussi concentrées localement.

La troisième situation renvoie à l'amorce d'une visibilité nationale ou internationale des entreprises. Les entreprises naissantes pourront alors accéder à des ressources convoitées et parfois géographiquement "éloignées" : les marchés. Dans de nombreux cas, les premiers marchés sont trouvés par l'intermédiaire des relais (géographiquement proches) mis en place lors de la structuration initiale de l'entreprise. Ainsi SYNTHETISEUR se créé dans un contexte où le fondateur "amène" des marchés antérieurement constitués. Pour VIOLON, l'un des premiers gros clients sera "amené" par l'un des principaux membres du collectif de création. Dans le cas de PIANO comme dans celui de XYLOPHONE (situations d'essaimage) la clientèle, qu'elle soit locale (industrie de l'aéronautique ou de l'espace) ou non, est déjà en contact avec les fondateurs. Dans le cas de VIOLONCELLE, le premier gros contrat obtenu transitera par le laboratoire auquel l'entreprise est adossée, qui le sous-traitera. Cela amènera la jeune entreprise à effectuer une mission d'expertise en Afrique. L'accès aux marchés est parfois lui-même conditionné par le partenariat avec des fournisseurs d'accès à des réseaux, numériques, électroniques ou téléphoniques, qui sont le plus souvent permis par le positionnement personnel et/ou professionnel de l'un des membres du collectif de création. En pratique plus les productions sont immatérielles (prestations de services, éditions logicielles, réseaux numériques) et plus les marchés peuvent être distants. Inversement, plus les productions sont matérielles, plus il y aura recherche d'une diversification des marchés tout en essayant de maintenir un ancrage sur les marchés locaux.

A un autre niveau, de nouvelles ressources peuvent être accessibles. Il s'agira notamment des finances telles que peuvent les amener des investisseurs institutionnels ou non. Ici, il ne s'agit plus des investissements initiaux, dont nous avons vu qu'ils étaient au départ massivement personnels et donc locaux. Ces investisseurs peuvent être identifiés à partir d'investisseurs institutionnels locaux, tels qu'un organisme régional de financement, qui contribue souvent activement au montage de tours de table financiers et qui peut identifier des partenaires financiers distants. Mais il peut également s'agir de «capitaux risqueurs » qui se positionnent spontanément vis-à-vis des entreprises (ce qui n'est par exemple pas rare dans le cas des entreprises de biotechnologies). Là encore, comme pour les marchés, on peut parfois constater l'existence d'une "distance" géographique entre les ressources recherchées et la localisation de l'entreprise.

Pour finir nous constatons qu'une troisième ressource est recherchée au travers des deux premières : il s'agit de la crédibilité, le plus souvent revendiquée au travers des "labels" affichés par exemple sur les différents site Web des jeunes entreprises. Une clientèle étrangère sera souvent présentée comme un signe de maturité de l'entreprise, l'existence d'investisseurs visibles comme un signe de confiance. De la même manière, les premières aides au montage 
de projet puis à la création d'entreprise, le fait d'avoir été lauréat de tel ou tel concours sont autant de signes qui seront présentés comme des marques de sérieux.

\section{La localisation des ressources et des formes de médiation}

Pour 39 de ces entreprises, nous avons codé les situations d'accès aux ressources ce qui permet de quantifier ce que nous avons analysé jusqu'à présent de façon qualitative. La mobilisation d'une ressource est une séquence d'action dans laquelle l'un des créateurs mobilise ou reçoit une ressource dont il ne dispose pas. Par exemple, la consultation d'un avocat pour rédiger les statuts est une séquence qui fait intervenir un ou plusieurs créateurs, l'avocat et les conseils que celui-ci dispense. L'avocat peut être une relation de l'un des créateurs ou une relation d'une relation, auquel cas nous considérons que la mobilisation de la ressource s'est effectuée par le biais des relations sociales, ou bien il peut avoir été choisi sur un annuaire, auquel cas nous considérons que c'est un dispositif de médiation qui a permis l'accès à la ressource. Le nombre de ces situations s'établit en moyenne à 15,1 par histoire (le minimum est 2 et le maximum 34), soit 590 en tout. Le nombre de situations varie selon la taille de l'entreprise (chaque recrutement est une ressource et donne lieu à une situation), la complexité de sa création, et la précision des informations que nous avons obtenues, mais les cas où nous avons véritablement rencontré des difficultés sont suffisamment rares pour que nous puissions considérer notre «échantillon » de situations comme correct, au moins pour l'objectif qui est le notre ici.

Le premier résultat est que les relations sociales occupent une place prépondérante dans les processus de création d'entreprises : $56 \%$ des situations codées font intervenir l'appel à des relations. Les relations sont plus fortement présentes dans la phase initiale, avant le dépôt des statuts (65\% des situations d'accès aux ressources font intervenir des relations). Les phases suivantes laissent plus de place aux ressources de médiation $(63 \%$ d'accès aux ressources par ces médiations lors de la deuxième année d'existence), ce qui illustre l'idée d'un découplage progressif de la nouvelle organisation par rapport aux relations de ses fondateurs. Tout se passe comme si l'organisation parvenait peu à peu à s'autonomiser vis-à-vis des relations des fondateurs, comme si l'encastrement initial, indispensable à sa création, s'affaiblissait progressivement au profit de logiques non relationnelles. Les relations mobilisées sont surtout professionnelles ( $80 \%$ des cas), mais assez souvent connotés affectivement (38\% de ces relations sont « amicalement professionnelles »).

Les autres modes d'accès aux ressources peuvent se diviser en deux catégories. La première est constituée par les organismes professionnels ou publics. Ce mode d'accès aux ressources est présent dans $21 \%$ des cas. La seconde catégorie, comprend des dispositifs de médiation, qui peuvent être des médias, divers médiateurs humains, les colloques, salons ou foire. Elle représente $23 \%$ des cas.

Examinons à présent le caractère plus ou moins local des ressources auxquelles les fondateurs d'entreprises accèdent par ces différents moyens. Dans le tableau qui suit, nous avons distingué un niveau local qui correspond à l'agglomération urbaine et un niveau «non local » qui regroupe tout ce qui est au-delà. 
Table 2. Type d'accès et localisation des ressources

\begin{tabular}{|l|c|c|c|c|}
\hline Type d'accès aux ressources & Réseaux & $\begin{array}{c}\text { Organisations } \\
\text { intermédiaires }\end{array}$ & $\begin{array}{c}\text { Ressources de } \\
\text { médiation }\end{array}$ & Total \\
\hline Localisation de la ressource & 251 & 98 & 87 & 436 \\
& $(58 \%)$ & $(23 \%)$ & $(20 \%)$ & $(100 \%)$ \\
& $(77 \%)$ & $(82 \%)$ & $(65 \%)$ & $14 \%)$ \\
\hline Non local & 75 & 22 & $(32 \%)$ & $(100 \%)$ \\
& $(52 \%)$ & $(15 \%)$ & $(35 \%)$ & $(25 \%)$ \\
\hline Total & $(23 \%)$ & $(18 \%)$ & 133 & 579 \\
& 326 & 120 & $(23 \%)$ & $(100 \%)$ \\
\hline
\end{tabular}

$($ Khi $2=10,1 ; p=0,06)$

Le premier constat est que les ressources sont très largement locales : 75\% dans la même agglomération, auxquels on pourrait ajouter $5 \%$ pour le reste de la région et encore $4 \%$ pour les régions proches.

Les relations sociales tendent à favoriser l'accès à des ressources locales, de même que les organisations intermédiaires, alors que les ressources de médiation sont un peu moins orientées vers les ressources locales. Le résultat est proche de celui d'une étude que nous avions menée sur les relations science-industrie (Grossetti et Bès, 2002), à ceci près que les organisations intermédiaires sont ici plus locales. Les ressources mobilisées sont massivement locales. Les clients sont le seul type de ressource à ne pas être majoritairement d'origine locale ( $45 \%$ seulement). Les plus locaux sont les conseils, qu'ils soient techniques $(88 \%)$ ou financiers, juridiques et marketing (86\%), les employés (88\% de recrutement local) et les fournisseurs $(78 \%)$. Si l'on tient compte du type de ressource, la corrélation entre le caractère local de celles-ci et l'accès par les relations est variable. Il se retourne même dans certains cas comme celui des locaux, où le passage par des médiations produit une localisation «locale » (en général en pépinière ou en incubateur), alors que dans le cas où les locaux sont trouvés par des relations, ils peuvent se situer à l'extérieur de l'agglomération. Les réseaux permettent d'accéder à des ressources plus locales (ils produisent de la proximité si l'on veut) dans le cas des employés, des clients et des fournisseurs. Les institutions sont plus «localistes » que les réseaux dans le cas des financements, des locaux et des conseils. Les dispositifs de médiation générique (le «marché » si l'on veut) permettent en général d'accéder à des ressources moins locales, sauf dans le cas des conseils, où existent manifestement des marchés locaux. A noter que, lorsque les fondateurs utilisent des relations sociales, la longueur des chaînes relationnelles mobilisées est en moyenne plus courte quand les ressources sont locales $(1,26$ contre 1,67 dans le cas où la ressource n'est pas locale). Le caractère plus ou moins local des ressources ne dépend pas du moment où celles-ci sont mobilisées (pas de «délocalisation » progressive de l'entreprise). Enfin, l'efficience des ressources est plus souvent faible lorsqu'elles ne sont pas locales et qu'elles ont été mobilisées par le marché.

\section{Conclusion}

La recherche présentée n'est pas achevée. Certains résultats doivent être confirmés ou affinés. Cependant, sur la base des données dont nous disposons d'ores et déjà, un certain nombre de 
points semblent clairs.

Le premier point concerne les choix de localisation. Lorsqu'il s'agit de déterminer la ville où l'entreprise sera créée, il n'y a en général tout simplement aucun choix : les fondateurs créent leur société là où ils sont en activité. Sauf dans quelques cas très particuliers, notamment lorsque les fondateurs sont dispersés dans l'espace, aucun raisonnement stratégique n'intervient. Alors même que des choix très élaborés sont effectués pour l'installation des familles, par arbitrage entre des contraintes et des enjeux multiples, la localisation des entreprises s'effectue le plus souvent selon une logique de l'évidence. Et, une fois la société installée, comme le remarque un chercheur allemand qui s'est intéressé à cette question, elle ne part pas (Stam (2006). Un quart des sociétés de notre échantillon on plus de 5 ans et aucune n'a changé de localisation. Par contre certaines ont créé des établissements ailleurs en France et surtout à l'étranger.

Pour comprendre le processus d'agglomération des entreprises innovantes, il faut donc se reporter aux conditions plus ou moins favorables à leur création. Comme nous l'avons vu, les ressources mobilisées sont très majoritairement locales, et celles-ci sont le plus souvent mobilisées par l'intermédiaire de relations personnelles ou de dispositifs locaux de médiation. C'est donc dans la répartition spatiale des ressources, les caractéristiques des réseaux, et dans l'activité des dispositifs locaux, qu'il faut rechercher les facteurs qui favorisent ou non la création d'entreprises innovantes. Les entreprises naissent plus facilement si les fondateurs peuvent accéder facilement à des ressources pertinentes. Lorsque ces ressources ne sont pas locales, il semble que les choses se passent mieux lorsque les créateurs y accèdent par l'intermédiaires de relations. Tout se passe comme si lorsqu'il n'y a pas de proximité spatiale, la proximité relationnelle puisse compenser le risque supplémentaire que peut constituer l'éloignement.

Les entreprises naissent dans une sorte de magma d'acteurs, de ressources et de relations, qu'elles structurent en retour. C'est la géographie de ce magma qu'il faudrait analyser pour comprendre les logiques spatiales de la création d'entreprises. Nous ne faisons là que retrouver des résultats classiques sur les milieux innovateurs, les districts, etc. Simplement, nous sommes allés bien plus loin que l'évocation de causes aussi vagues et insaisissables que l' « atmosphère industrielle », la «culture locale » ou des «milieux », en nous donnant les moyens d'une description plus rigoureuse de ce qui se cache derrière ces désignations. Notre compréhension des logiques spatiales en devient un peu meilleure.

\section{Références}

Granovetter Mark S., 1985, "Economic action and social structure : the problem of embeddedness", American Journal of Sociology, Vol. 91, n³, pp.481-510.

Grossetti Michel, Barthe Jean-François, Beslay Christophe, 2006, « La mobilisation des relations sociales dans les processus de création d'entreprises. Aperçus à partir d'une enquête en cours ». Sociologie pratique, Avril. 
Grossetti Michel, 2004, « Concentration d'entreprises et innovation : Esquisse d'une typologie des systèmes productifs locaux », Géographie, économie, société, 2004, Vol. 6, n², pp : 163177.

Grossetti Michel et Bès Marie-Pierre, 2002, "Proximité spatiale et relations scienceindustrie: savoirs tacites ou encastrement (Polanyi ou Polanyi)», Revue d'Economie Régionale et Urbaine, ${ }^{\circ} 5$ - II -, pp.777-788.

Powell Walter and Smith-Doerr Laurel, 1994, "Networks and économic life", in Neil J. Smelser and Richard Swedberg (eds), The Handbook of economic sociology, Princeton, Russel Sage Foundation, pp.368-402.

Stam Erik, 2006, «Why butterflies don't leave. Locational behaviour or entrepreneurial firms », The papers on entrepreneurship, growth and public policy, MPI Jena, Max Planck Institute of Economics.

Zucker, L., Darby, M., Armstrong, J., Intellectual Human Capital and the Firm: The Technology of Geographically Localized Knowledge Spillovers, NBER Working Paper N0. 4946, 1994. 